\title{
Dynamic Queue and TCP based Multipath Congestion Control Scheme for Wired Network
}

\author{
Dinesh Gupta \\ Scholar, M.Tech \\ Department of Computer Science \\ TIT, Bhopal, India
}

\author{
Deepak Singh Tomar \\ Asst. Professor \\ Department of Computer Science \\ TIT, Bhopal, India
}

\begin{abstract}
High speed wired communication are large bandwidth utilized network but shared by multiple sender that is crucial challenge for congestion control for that types of network. This paper identifies that dynamic queue and multipath based routing strategies for wired networks are more suitable for congestion control and memory management as compared to existing queue based congestion control, paper describe about existing queue techniques that is RED, Drop-tail etc. and its result impact than compare their results with proposed dynamic base multipath routing in wired network scenario and conclude that in proposed mechanism better bandwidth utilized and control the congestion as well as data drop in multiple sender environment. The results retrieved from network simulator-2 and analyzed the performance of packet delivery ratio, throughput, TCP and UDP data sends and receives in proposed environment deployed based.
\end{abstract}

\section{Keywords}

TCP, UDP, Wired Communication, Drop-tail, RED, Queue base Drop, PDR, Packet Drop, Throughput

\section{INTRODUCTION}

Different application over internet require high transfer data rate as the demand increases. These applications run concurrently and utilize the network resources at the same time thus creating problems like congestion. Data transfer over internet takes place using most efficient TCP protocol; it controls network congestion by efficiently measuring packet loss rate. Congestion must be controlled in modern internet scenario to avoid packet loss. Internet users are transferring large volume of data every day. Congestion problem occurred when arrival rate to a particular router is more than its departure rate. Every router uses Active Queue Management (AQM) [1] and scheduling for congestion control. Queue management system is designed to control various types of queues. Queuing theory is a process for queue creation and propagation. The AQM algorithms effort to control the length of packet queues by dropping packets whenever required to control congestion. On the other hand scheduling algorithms has a role to determine next packet drop and which packet needs to be sent and it allocates bandwidth among flows. At routers, buffer management scheme decides dropping policy. Drop-tail is the simplest queue management scheme. In Drop tail scheme each packet is treated identically. When the queue is filled to its full size, the newly arriving packets are dropped until the queue has adequate room to accept arriving traffic. As queue has been filled, the router initiates discarding all further datagram's, thereby dropping the tail of arrangement of datagram. TCP sender now process slow start.

\section{RELATED WORK}

The research work done previously in this field is presented in this section.

Researcher has worked on a technique using multipath distance vector routing and ranging queue base theme for congestion minimization, thus terribly first we tend to find numerous congestion occurrence in network after that apply multipath routing along with varied queue approach that method reduce the congestion and improve the network performance. We design proposed algorithm and proposed work and the performance is evaluated and simulate through all network parameters [2].

Author has done research on Standard RED as base to evaluate the performance of RED with ECN. Standard RED has the ability to control congestion by reducing the packet drop rate. TCP, FTP and Constant Bit Rate (CBR) traffic based on UDP are used to assess the performance. Although ECN perform well on packets drops but it does not necessarily able to improve throughput for TCP transfers. In summary, the TCP/IP ECN sender responds better than the ECN unaware sender by reducing congestion and can avoid failed segment transmissions. Nevertheless, TCP/IP ECN and standard TCP have the same mechanisms for detecting better quality network conditions and therefore, are unable to deliver guaranteed improved result [3].

Author has worked on the efficiency and the performance of TCP in case of persistent congestion and result shows that efficiency of TCP using ECN increases without spoiling its performance. AQM techniques and Random Early Detection (RED) has been in concern as their capability to perform well with bottleneck links. Experimental study of RED, Drop-tail and RED plus ECN (Explicit Congestion Notification) when functioning over a frequently congested link is done in this paper [4].

Author has proposed a simple variation to the drop tail algorithm in which a genuine queue management scheme controls congestion in TCP/IP networks. In that case we dynamically adjust queue length in our wired network. Adjusting queue length (varying queue to maximum range for drop tail) while the buffer size of the input port is fix gives the good performance. The proposed scheme shows the great enhancements as compare to drop tail, and RED algorithm in terms of packet loss rate ,and buffer utilization in different network environment (such as topologies and traffic condition) [5].

Author has proposed multipath and dynamic queuing based load balancing approach in Data centre networks. Varying queue scheduling methods improves overall quality of service in case of congestion [6]. 
Researcher worked on delay \& jitter based unidirectional TFRC along with end-to-end semantic for wired and wireless networks. This technique provide improved transfer rate and TCP pleasant features like standard TFRC, besides bursts nature of background traffic it also enhance the throughput by evaluating the available bandwidth in wired-wireless networks. Simulation results show great improvement in performance without intrusiveness issue and besides bursts background traffic over wired-wireless networks [7]

Researcher extends AQM mechanism from single network systems area to large-scale wired network systems along with time delays at every communication channel. A system model contained of numerous local networks is first created. Lyapunov stability theory is used to drive stability condition guaranteeing whole stability. Stability of large- scale wired networks using RED as AQM strategy for queue is modeled and evaluated in this research. Feedback control problem has been fixed for the LWNS with delay in the interconnections and whole loop stability is achieved in new condition [8].

Author has explained the traditional queuing technique used to control traffic congestion. Drop-tail tends to penalize bursts links by dropping all the received packets only when the buffer is filled. This will continue until the no. of packets in the queue become less than maximum queue length and congestion is removed. This method has two main drawbacks: 'Lock-Out': result of global synchronization, and 'Full Queue': results in high packet delay [9].

Researchers have investigated mechanism for internet congestion control and RED model as a feedback system. RED model was used to handle traffic dynamics (flows) in TCP/IP network. RED parameters are implemented as a configuration program that can be used by network for router's configuration [10]

Author has proposed Random early detection technique to avoid congestion before its occurrence. RED technique marks every packet that arrives at the router as the average queue size surpasses a maximum threshold. If RED router marks packets by dropping them, when the average queue size surpasses the maximum threshold, then the RED router controls the average queue size besides the absence of cooperating transport protocol [11].

Author discusses some of the deficiencies of Random Drop and Drop Tail gateways, and investigates Early Random Drop gateways. In this work, Implementation of Early Random Drop, if the queue length surpasses a certain drop level, then the gateway drops next packet arriving at the gateway with certain drop probability [12]

Author has proposed a rate based congestion control method that relies on bandwidth estimation tool. Bandwidth estimation tool can achieve better channel efficiency over TCP. Bandwidth aware congestion control helps to increase performance and link utilization [13].

Author studies the performance of various Active Queue Management schemes in both wired as well as wireless networks based on the TCP flow. In Active Queue Management Which scheme performs well when congestion occurs in wired and wireless networks. Performance evaluation of Drop-tail, Random Early Detection (RED, Random Exponential Marking (REM) carried out [14].

Author has worked on queue, delay and loss characteristics of various Active Queue Management schemes in QoS-enabled network. PI scheme and Feedback-based mechanism in delay sensitive applications is described. Each methods design goal, performance analysed [15].

Author has analysed the comparison of various queuing techniques to determine the best solution to avoid congestion in networks. Comparison of RED, BLUE, REM and SQF algorithms is carried out. Which queuing algorithm should be used in a condition is described in detail [16]

\section{PROBLEM STATEMENT}

Congestion occurs in data networks when transfer requests exceed the actual overall capacity of the nodes or routers in network. This may render into poor quality of service for the user. It may also result in packet loss, end to end delay or blockage of new connections. The problem of congestion control is more difficult to handle in networks with connectionless protocols like UDP, than in with connectionoriented protocols like TCP. In connection-oriented TCP networks, resources in the network are reserved in advance during connection setup. Thus, one easy way to control congestion is to prevent new connections from starting up if congestion is sensed. The disadvantage is that if any other reservation scheme is that reserved resources may not be used and may be left idle even when other users have been denied permission. In this paper we focus number of different congestion control using queue system and all are feasible their own point of view for TCP transmissions but we further design dynamic drop tail queue mechanism and provide fairly serve TCP as well as UDP data under wired communication.

Because of following reasons:-

- Bandwidth constraint is limited.

- Buffer capacity of intermediate nodes is Limited

- Multipath routing procedures are absent.

- Efficient load balancing means that evenly distribute load at each node on the network properly.

- Unsteady packet delivery, end to end delays and packet losses in case of link failure.

\section{PROPOSED WORK}

In our proposal we minimize data drop through acknowledgement delay estimation base rate selection as well as dynamic queue base drop minimization, very first we set initial parameter of wired and wireless communication and after that we select best possible multiple path from source to destination, and then we send data, in that case we apply TCP New Reno updated technique and calculate acknowledgement delay difference and set new data size for transmission the data but if incoming rate is faster than the outgoing rate then we apply dynamic queue scheme for minimization data drop from the network TCP New Reno are alert the sender for slow your transmission.

\subsection{Algorithm}

$\mathrm{N}:\left\{\mathrm{n}_{1}, \mathrm{n}_{2},-------\mathrm{n}_{\mathrm{n}}\right\}$ set of nodes

$\mathrm{L}:\left\{1_{1}, 1_{2}, \quad 1_{m}\right\}$ link between nodes

$1_{m}=n_{i} \leftarrow \rightarrow n_{j} \quad$ where $n_{i}, n_{j}$ is node

$\mathrm{L}_{\mathrm{b}}$ : link bandwidth

$\mathrm{Q}:\left(\mathrm{q}_{1}, \mathrm{q}_{2, \ldots \ldots \ldots} \mathrm{q}_{\mathrm{k}}\right\}$ queue in link

S: sender node

$\mathrm{R}$ : receiver node

Rt_Proto $=$ DV : distance vector

S-established the multipath using DV proto

If (path ${ }_{1}$ and path ${ }_{2}$ is available) 
Calculate bandwidth

Send bandwidth info and shared node to sender

Send data through all available paths

receives $\mathrm{msg}$

Generate CBR pkts

While $\left(\mathrm{n}_{\mathrm{j}}\right.$ share $\mathrm{l}_{\mathrm{i}}$ link \&\& $\mathrm{l}_{\mathrm{i}}$ occupy by $\mathrm{S}$ node $)$

$\mathrm{n}_{\mathrm{j}}$ generate data on the bases of available bandwidth and $\mathrm{q}_{\mathrm{i}}$

if $\left(n_{k}\right.$ demand the link $1_{i} \& \& 1_{i}$ busy \&\& $q_{i}$ utilized by $\left.S\right)$

\{

Set new $\mathrm{q}_{\mathrm{k}}$ for $\mathrm{n}_{\mathrm{k}}$ data

Send $\mathrm{l}_{\mathrm{i}}$ available bandwidth info to $\mathrm{n}_{\mathrm{k}}$

$\mathrm{n}_{\mathrm{k}}$ set new data rate

If (bandwidth utilized $>50 \% \& \& \mathrm{q}_{\mathrm{i}}<10 \& \& \mathrm{q}_{\mathrm{k}}>10$ )

$\mathrm{q}_{\mathrm{i}}$ decr

$\mathrm{q}_{\mathrm{k}}$ incr

\}

Else

No change in queue size

\}

\}

If (congestion occurs in $\mathrm{n}_{\mathrm{i}}$ )

$\mathrm{N}_{\mathrm{i}}$ send msg to $\mathrm{n}_{k}$ senders for minimized the data rate

$\mathrm{S}$ change data rate

Free (congestion)

Analysis the network behavior PDR, throughput, total send and receives. \}

\section{SIMULATION ENVIRONMENT AND RESULTS}

The Network Simulator (Version 2), widely known as NS2, is simply an event driven simulation tool that has proved useful in studying the dynamic nature of communication networks. Simulation of wired, wireless as well as ad hoc network functions and protocols like TCP, UDP etc. can be done using NS2. In general, NS2 provides users with a tactic of specifying such network protocols and simulating their corresponding performances. Due to its flexibility and modular in nature, NS2 has gained constant acceptance in the networking research community since its entrance in 1989. Ever since, several revolutions and modifications have marked the growing maturity of the tool, thanks to substantial assistances from the players in the field. Among these are the University of Cornell and Cornell California University who developed the REAL network simulator, 1 the foundation on which NS is based. Since 1995, Defense Advanced Research Projects Agency (DARPA) supported development of NS through the Virtual Inter Network Test bed (VINT) project [17]. Currently the National Science Foundation (NSF) has joined the ride in development. The group of researchers and developers in the community are constantly working to keep NS2 strong and versatile.

\subsection{Performance Metrics}

We are taking the following parameters for case study i.e. shown in Table 1 .
Table 1 Simulation parameter

\begin{tabular}{|l|l|}
\hline $\begin{array}{l}\text { Number of } \\
\text { nodes }\end{array}$ & 17 \\
\hline $\begin{array}{l}\text { Routing } \\
\text { Protocol }\end{array}$ & DV \\
\hline $\begin{array}{l}\text { Simulation } \\
\text { time (seconds) }\end{array}$ & 100 \\
\hline Queue Type & $\begin{array}{l}\text { Drop-tail, RED and DQ-Drop } \\
\text { tail }\end{array}$ \\
\hline Queue Length & 20 (dynamic if required more) \\
\hline $\begin{array}{l}\text { Transport } \\
\text { Layer }\end{array}$ & TCP (TCP New reno),UDP \\
\hline Traffic type & CBR, FTP \\
\hline $\begin{array}{l}\text { Packet size } \\
\text { (bytes) }\end{array}$ & 1000 \\
\hline
\end{tabular}

\subsection{Results}

In this section we examine the results analysis on the basis of performance metrics and different drop reasons.

\subsubsection{Packet delivery Fraction Analysis}

It is the ratio of data packets delivered to the destination to those generated by the sources.

It is calculated via dividing the number of packet received by destination through the number packet originated from source.

$$
\mathrm{PDF}=(\mathrm{Pr} / \mathrm{Ps}) * 100
$$

Where Pr is total Packet received \& Ps is the total Packet sent. In wired communication simulation that performance is depends on the characteristic of wired medium and queue capacity of the link.

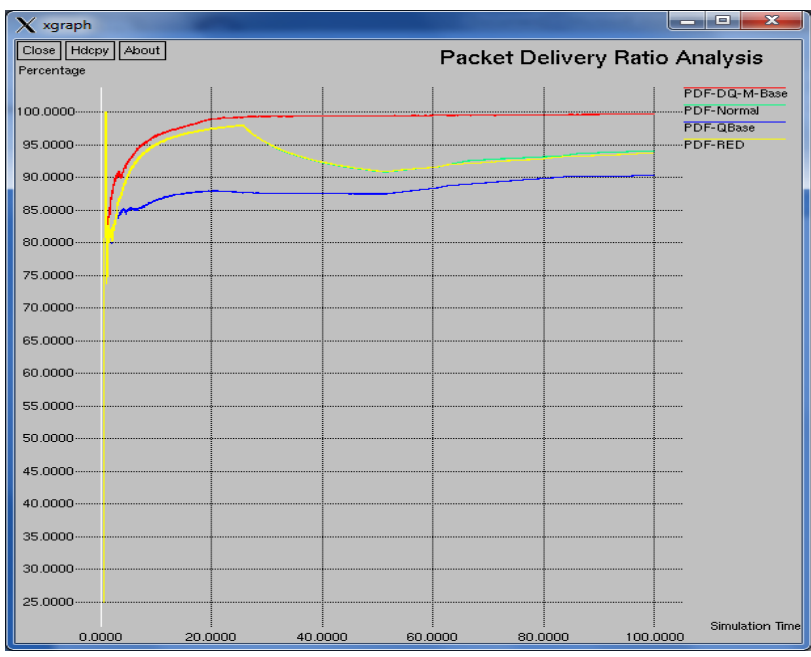

Figure 1: Packet delivery Fraction Analysis

Graph shows (see Figure 1) the result of packet delivery ratio under the existing queue technique with multipath routing and single path and compare with proposed dynamic queue multipath routing technique and $\mathrm{x}$ axis shows the simulation time in second and $y$ axis shows percentage of data delivery in each cases, here easily identifies that our proposed 
dynamic queue perform outstanding as compare to existing methods.

\subsubsection{Throughput Analysis}

The packets receiving measured in network in per unit of time are evaluated during simulation are called throughput.

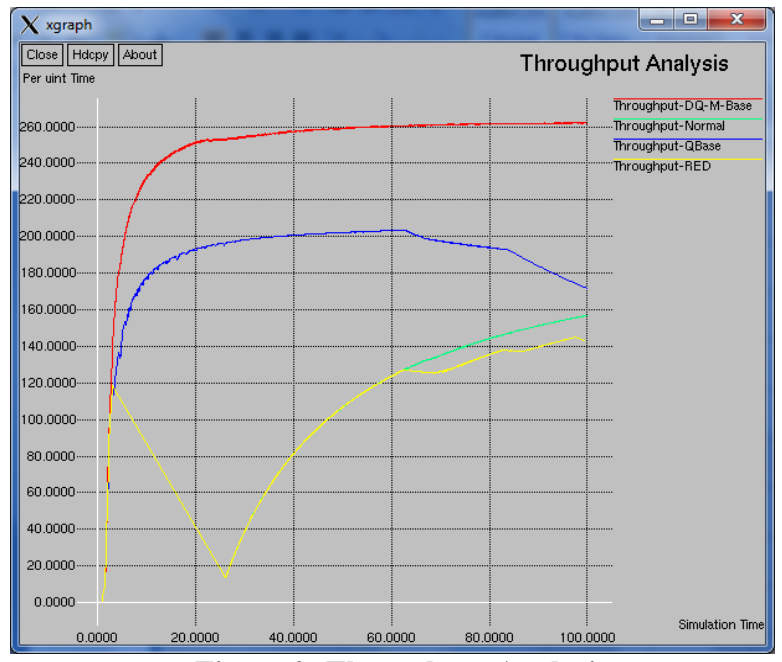

Figure 2: Throughput Analysis

This parameter proportional the packet delivery ratio, in the simulation environment takes two queue techniques and analyzes the behavior under single path and multipath routing and identifies that random early detection queue (RED) gives less performance rather all other module and Proposed dynamic queuing with multipath routing shows (see Figure 2) better perform in all the time that is nearly 260 packets per seconds.

\subsubsection{TCP Data Transmission Analysis}

Transmission control protocol is acknowledgment based data delivery method and more reliable as compare to UDP. During the simulation we create the multiple TCP links and analyze the total transmission by the transmitter in all cases.

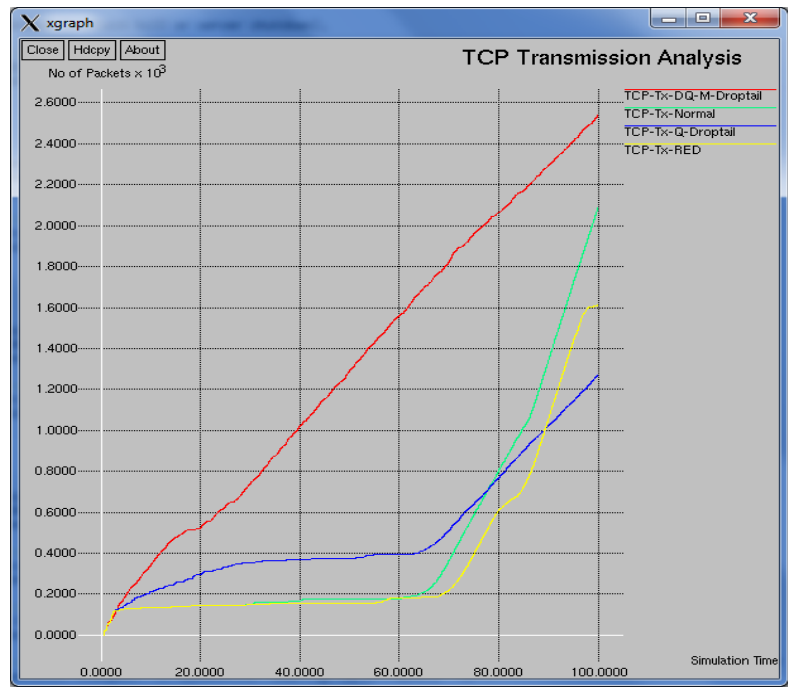

Figure 3: TCP Data Transmission Analysis

Graph shows (see Figure 3) that the analysis methodology apply all four method in similar way and where proposed multipath routing with dynamic queue inbuilt in module than number of TCP transmission are increases because data are simultaneously send by the multiple path. Dynamic queue technique suits well in wired network.

\subsubsection{TCP Data Receives Analysis}

Transmission Control Protocol (TCP) is an end to end delivery protocol that provides the connection oriented technique to deliver the data in between sender and receiver. The connection oriented technique of data transmission is time consuming because of establishment of connection as well as acknowledgement receives by the sender are increased waiting time rather than UDP technique.

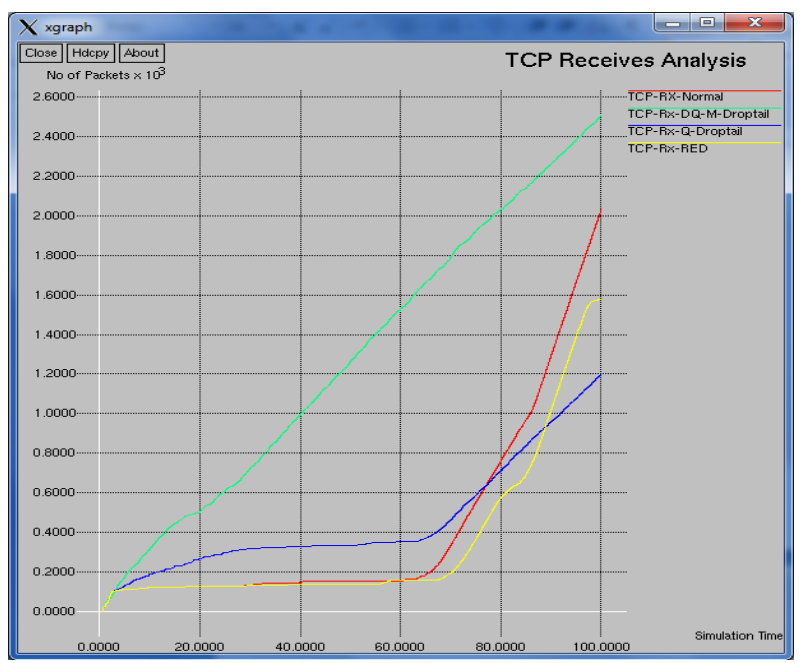

Figure 4: TCP Data Receives Analysis

Graph shows (see Figure 4) the result of TCP data receiving by the authorized receiver in all cases and conclude the graph proposed multipath routing and dynamic queue approach are maximum data receives as compare to all existing methods.

\subsubsection{TCP Data Lost Analysis}

Data lost are depends on the network behavior like available bandwidth, congestion, collision, queue, interruption, interference etc.

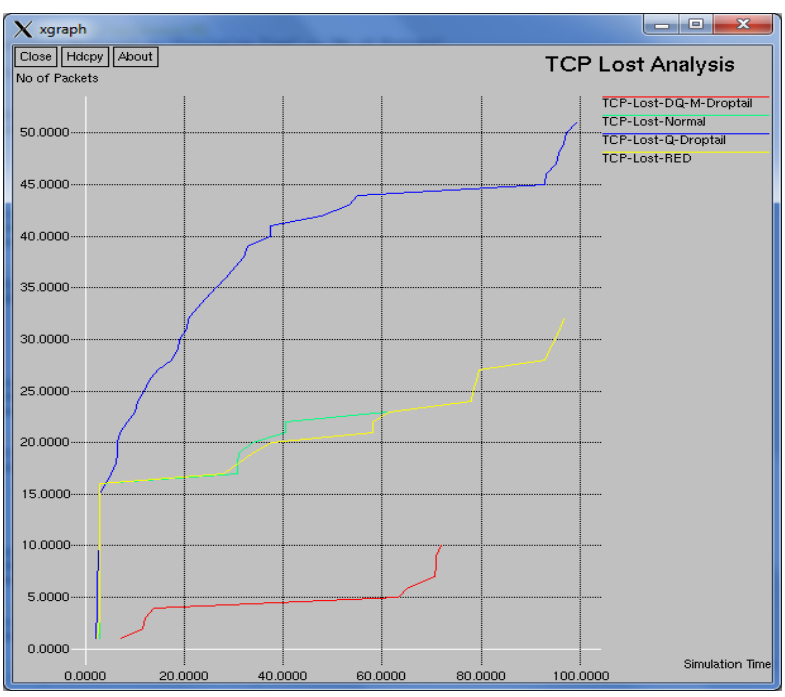

Figure 5: TCP Data Lost Analysis

Graph shows (see Figure 5) the analyzed TCP data lost in all four condition and summarized that, proposed methodology has minimum lost as compare to all existing technique because proposed case dynamical varies the queue while 
sender need the more queue in intermediate node then on the demand bases queue has increases and save the data from lost but other existing approach we can't predict or increase in on demand base so data lost are greater in case of existing methodology.

\subsubsection{UDP Data Lost Analysis}

In this graph shows the UDP data lost in wired communication structure in single path and multipath routing case, that user datagram protocol are fast data service but not reliable because it's not provide the guaranty of the data delivery. So that totally depends on the network behavior, while no any obstacle in between communication, queue and bandwidth are available than minimum data.

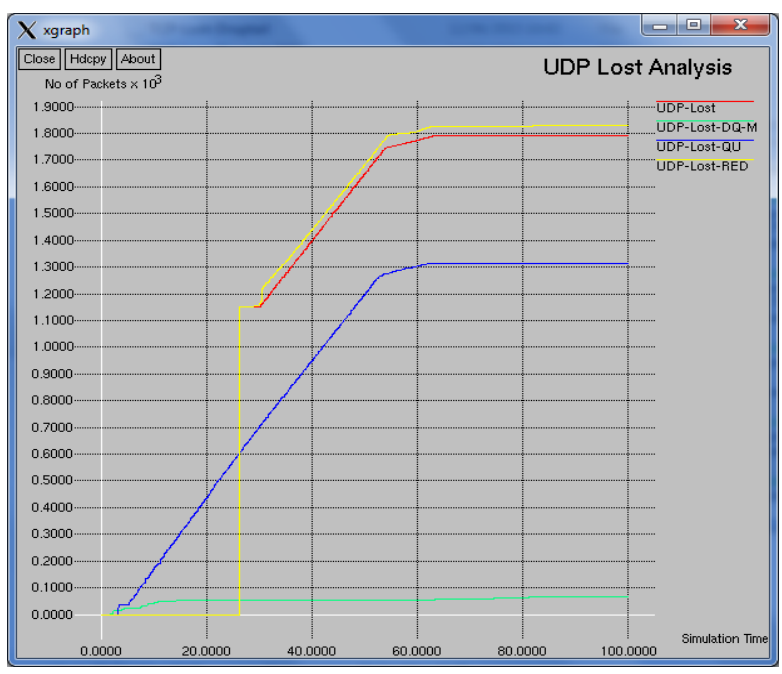

Figure 6: UDP Data Lost Analysis

Graph shows (see Figure 6) that minimum data lost while proposed methodology apply that is nearly $0-10$ packets and all other existing methods are not control from the huge amount of data lost.

\subsubsection{UDP Received Analysis}

User Datagram Protocol (UDP) is the Transport layer protocol that works on connection less mechanism.

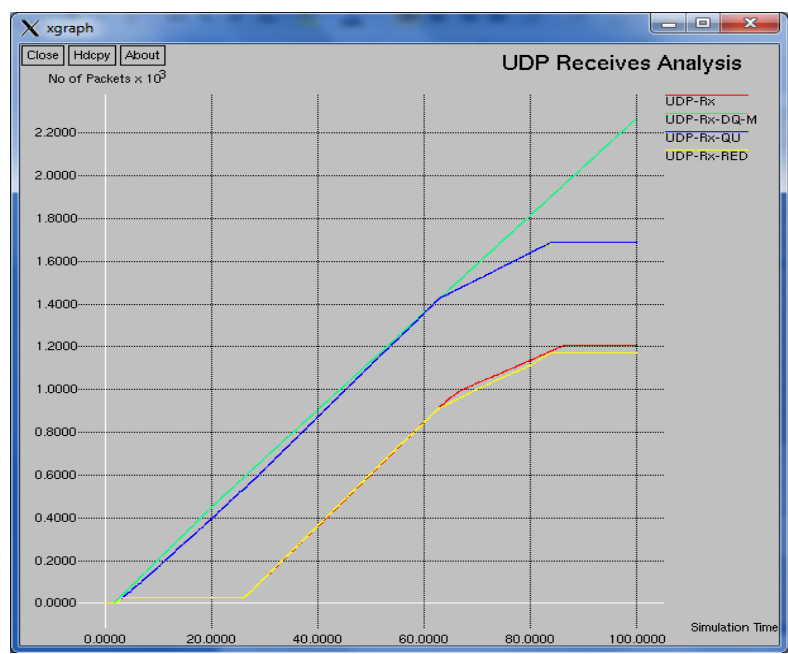

Figure 7: UDP Received Analysis

It means no Acknowledgement (ACK) from receiver is send back to sender of successful data delivery. Graph $\mathrm{x}$ axis shows (see Figure 7) the simulation time and y axis shows the number of UDP packets successfully receives, in the dynamic queue with multipath (proposed) time packets receiving is nearly 2300 and rest of exiting methodology time that receiving is lower that the proposed method.

\subsubsection{Overall Performance Summary of old and Proposed Scheme}

The overall analysis in case of old scheme and proposed scheme are mentioned in Table 2. Here we noticeably observed the performance of proposed scheme is improves the routing mechanism and represents better results than old scheme.

Table 2: Analysis Summary

\begin{tabular}{|c|c|c|c|c|}
\hline \multicolumn{5}{|c|}{ Overall Summary } \\
\hline Parameter & Unipath & $\begin{array}{c}\text { Q- } \\
\text { Drop- } \\
\text { Unipath }\end{array}$ & $\begin{array}{c}\text { RED } \\
\text { Based }\end{array}$ & $\begin{array}{l}\text { Proposed } \\
\text { DQ- } \\
\text { Multipath }\end{array}$ \\
\hline Packet Sent & 12378 & 14287 & 11834 & 14186 \\
\hline $\begin{array}{c}\text { Packet } \\
\text { Received }\end{array}$ & 11651 & 12905 & 11093 & 14147 \\
\hline PDF & 94.13 & 90.33 & 93.74 & 99.73 \\
\hline $\begin{array}{l}\text { Average e- } \\
\text { e delay(ms) }\end{array}$ & 1198.46 & 1153.52 & 650.01 & 1207.27 \\
\hline $\begin{array}{c}\text { No. of } \\
\text { dropped } \\
\text { data } \\
\text { (packets) }\end{array}$ & 727 & 1382 & 741 & 39 \\
\hline
\end{tabular}

\section{CONCLUSION}

The wired network is more reliable then the wireless network because of data is flow from guided media but their cost as compare to wireless is more. In this research we proposed the dynamic queue length technique with multipath concept is applying to reduce the possibility of packets loss and multipath routing is reduces the possibility of congestion in a particular link or path in a wired network. The simulation of Drop-tail queue scheme and RED scheme is done with unipath routing protocol. In Drop-tail packet is allowed to enter in queue till the queue is empty and drop the entire incoming packets when queue becomes full. In Drop-tail queue model there is no any approach for early detection of congestion before queue become overflow i.e. in congested network packet drop is common problem and re-transmission of all dropped data packets will consume lot of resources such as transmission link capacity and processing power of nodes. The RED (Random Early Detection) algorithm queue model is based on the fixed minimum and maximum threshold value. The data packets are cross the limit of threshold in that case the probability of data dropping is increases. The simulation results are shows the performance of proposed dynamic queue variation with multipath routing is improves the network performance reduces the packet loss, reduces routing overhead, enhance network throughput and Packet Delivery Frequency as compare to Drop-tail and RED Queue model.

The concept of queue length variation is also possible in different network like VPN (Virtual Private Network). In VPN network the separation of collision domain is possible it implies that only some ports starts rest of them are not accessible. In future we applying the proposed scheme in 
VPN network and compare the performance with this dissertation research work.

\section{REFERENCES}

[1] Adms R. 2012 "Active Queue Management: Survey" IEEE Communications Surveys \& Tutorials, IEEE ISSN :1553-877X ,Volume:15, Issue: 3 Page(s):1425 - 1476 11.

[2] Hitesh Gupta, Pankaj Pandey 2013 "Congestion Control using Varying Queue Base Approach as Well as Multipath Routing” (IJERT) Vol. 2 Issue 12.

[3] Alireza Gharegozi 2010 "The Study of ECN Application Effect at the Performance Improvement of RED" IEEE 2nd International Conference on Computer Technology and Development (ICCTD 2010), pp. 632-636.

[4] Stefanos Harhalakis, Nikolaos Samaras and Vasileios Vitsas 2011 "An experimental study of the efficiency of Explicit Congestion Notification", IEEE Panhellenic Conference on Informatics, pp. 122-126.

[5] Manoj Kumar Prajapati, Divakar Singh 2012 "Verifying Queue Length Scheme in Wired Communication for Congestion Control" Scholars Research Library European Journal of Applied Engineering and Scientific Research, 1 PP.91-98.

[6] Sadhana Gotiya, Nitin Mishra 2014 "Multipath and Dynamic Queuing base load balancing in Data Centre Network" IJACT Vol 3 issue 6.

[7] Amit Kumar, R.M.Sharma 2012 "A New Congestion Control Approach on TFRC Over Wired and Wireless Networks", IOSR Journal of Computer Engineering (IOSRJCE) ISSN: 2278-0661 Volume 1, Issue 4, pp. 0108 .

[8] Ping-Min Hsu, Chun-Liang Lin, Ching-Han Yu 2011 "Active Queue Management within Large-Scale Wired
Networks", Int. J. Communications, Network and System Sciences(scientific research), 4, 241-248.

[9] B. Braden, D. Clark, J. Crowcroft, B. Davie, S. Deering, D. Estrin, S. Floyd, V. Jacobson, G. Minshall, C. Partridge, L. Peterson, K. Ramakrishnan, S. Shenker, J. Wroclawski, and L. Zhang 1998 "Recommendations on Queue Management and Congestion Avoidance in the Internet," RFC 2309.

[10] Victor Firoiu Marty Borden 2000 "A Study of Active Queue Management for Congestion Control” In IEEE INFOCOM.

[11] Sally floyd and Van Jacobson 1993 "Random early detection gateway for congestion avoidance" August 1993 IEEE /ACM transaction on networking.

[12] Hashem, E. 1989 "Analysis of random drop for gateway congestion control”, Report LCS TR-465, Laboratory for Computer Science, MIT, Cambridge, MA, p.103.

[13] Muhammad Choudhury, Khaled Harfoush 2012 "Internet Congestion Control with network measurement support" IEEE 978-1-4673-2890-6/12.

[14] Abdullah Al Masud, Hossain Md, Shamim, Amina Akhter 2011 "Performance Analysis of AQM Schemes in Wired and Wireless Networks based on TCP flow" IJSCE Vol. 1 issue-3.

[15] Jahwan Koo, Seongjin Ahn, Jinwook Chung 2015 “A comparative study of queue, delay, and loss characteristics of AQM schemes in QoS-enabled networks" Computing and Informatics, Vol. 22, 317-335.

[16] Sahil Kochher, Gurnam Singh 2014 "Comparison of Various Queuing Techniques to Select Best Solution for Eradicating Congestion in Networks" IJECS Vol 3, page 7010-1017.

[17] The Network Simulator - ns-2. [Online]. Available: http://www.isi.edu/nsnam/ns/ 